\title{
autoplotly: An R package for automatic generation of interactive visualizations for statistical results
}

DOI: $10.21105 /$ joss. 00657

\section{Software}

- Review ¿

- Repository ca

- Archive ca

Submitted: 20 March 2018

Published: 19 April 2018

\section{Licence}

Authors of papers retain copyright and release the work under a Creative Commons Attribution 4.0 International License (CC-BY).

\section{Yuan Tang ${ }^{1}$}

$1 \mathrm{H} 2 \mathrm{O}$. ai

\section{Summary}

Often times users only want to quickly iterate the process of exploring data, building statistical models, and visualizing the model results, especially the models that focus on common tasks such as clustering and time series analysis. Some of these packages provide default visualizations using base R's plot () for the data and models they generate. However, they look out-of-fashion and these components require additional transformation and clean-up before using them in ggplot2 (Wickham 2009) and each of those transformation steps must be replicated by others when they wish to produce similar charts in their analyses. Creating a central repository for common/popular transformations and default plotting idioms would reduce the amount of effort needed by all to create compelling, consistent and informative charts. The ggfortify (Tang, Horikoshi, and Li 2016; Horikoshi and Tang 2018) package provides a unified ggplot2 plotting interface autoplot() to many statistics and machine learning packages and functions in order to help these users achieve reproducibility goals with minimal effort. ggfortify package has a very easy-to-use and uniform programming interface that enables users to use one line of code to visualize statistical results of many popular $\mathrm{R}$ packages using ggplot2 as building blocks. This helps statisticians, data scientists, and researchers avoid both repetitive work and the need to identify the correct ggplot2 syntax to achieve what they need. Users are able to generate beautiful visualizations of their statistical results produced by popular packages with minimal effort. plotly (Sievert et al. 2018) is an R package built on top of Javascript visualization framework plotly.js that become popular building blocks for creating interactive visualizations in R. Its ggplotly() function can convert graphics created by ggplot2 to interactive plots with plotly style.

The autoplotly (Tang 2018b, 2018a) package is an extension built on top of $\mathrm{R}$ packages ggplot2, plotly, and ggfortify to provide a unified autoplotly() function to automatically generate interactive visualizations for many popular statistical results supported by ggfortify package, such as time series, PCA, clustering and survival analysis, with plotly and ggplot 2 style. The generated visualizations can also be easily extended using ggplot2 and plotly syntax while staying interactive.

\section{References}

Horikoshi, Masaaki, and Yuan Tang. 2018. Ggfortify: Data Visualization Tools for Statistical Analysis Results. http://CRAN.R-project.org/package=ggfortify.

Sievert, Carson, Chris Parmer, Toby Hocking, Scott Chamberlain, Karthik Ram, Marianne Corvellec, and Pedro Despouy. 2018. Plotly: Create Interactive Web Graphics via 'Plotly.js'. 
Tang, Yuan. 2018a. "Autoplotly - Automatic Generation of Interactive Visualizations for Popular Statistical Results." arXiv Preprint arXiv:1803.08424.

2018b. Autoplotly: Automatic Generation of Interactive Visualizations for Popular Statistical Results. https://github.com/terrytangyuan/autoplotly.

Tang, Yuan, Masaaki Horikoshi, and Wenxuan Li. 2016. "Ggfortify: Unified Interface to Visualize Statistical Result of Popular R Packages." The $R$ Journal 8 (2):478-89. https://journal.r-project.org/.

Wickham, Hadley. 2009. Ggplot2: Elegant Graphics for Data Analysis. Springer Science \& Business Media. https://doi.org/10.1007/978-0-387-98141-3. 